Notre Dame Journal of Formal Logic

Volume XIV, Number 1, January 1973

NDJFAM

\title{
A NOTE ON NEWMAN'S ALGEBRAIC SYSTEMS
}

\author{
BOLESŁAW SOBOCIŃSKI
}

This note possesses a purely supplementary and informative character with respect to the papers [2], [3], [4], [5] and [6]. ${ }^{1}$ Namely, in order to describe the systems investigated in those papers more completely the definitions of the dual associative Newman algebras which are mentioned only casually in [6], p. 536, and of the dual mixed associative Newman algebras will be established. Additionally, a rather bad misprint and erroneous statement which both appear in [3] will be corrected.

1 It has been established in [4] that the associative Newman algebras can be defined, as follows:

Any algebraic structure

$$
D=\langle A,+, \times,-\rangle
$$

where + and $\times$ are two binary operations, and - is a unary operation defined on the carrier set $A$, is an associative Newman algebra, if it satisfies the following postulates:

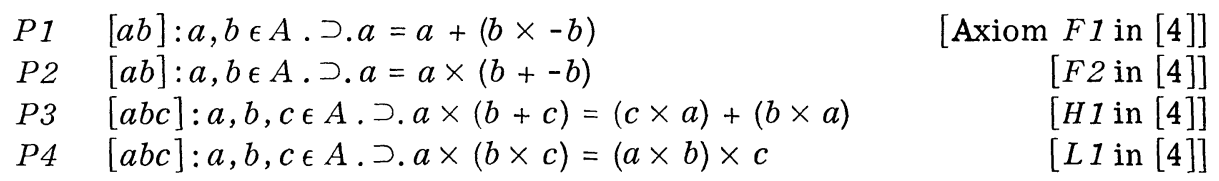

Therefore, it is self-evident that the dual associative Newman algebras can be defined as follows:

Any algebraic structure

$$
\mathfrak{\Re}=\langle A,+, \times,-\rangle
$$

1. An acquaintance with the papers [2]-[6] is presupposed. Concerning the symbols used in this note it should be remarked that instead of " $\bar{a}$ ", which is used in [2], [3] and [4] I am using here " $-a$ ". An enumeration of the algebraic tables, $c f$. section 3 below, is a continuation of the enumeration of such tables given in [2], $[4],[5]$ and [6]. 
where + and $\times$ are two binary operations, and - is a unary operation defined on the carrier set $A$, is a dual associative Newman algebra, if it satisfies the following postulates:

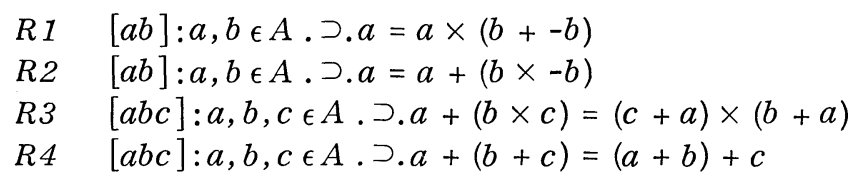

Since fl $^{1}$, $c f$. [6], p. 542, verifies the axioms $R 1-R 4$, but falsifies the law of idempotency with respect to the operation $\times$, we know that system $\mathfrak{R}$ is not necessarily a Boolean algebra. In section 3 , point (1), below the mutual independency of the postulates $R 1-R 4$ will be proved.

Using the deductions entirely analogous to those which are given in [4] we can prove easily that in the field of the fixed carrier set $A$ the axioms $R 1-R 4$ are inferentially equivalent to the following formulas: $R 1, R 2, R 4$ and

R5 [ab]: $a, b \in A . \supset . a+b=b+a$

R6 $[a b c]: a, b, c \in A . \supset . a+(b \times c)=(a+b) \times(a+c)$

and, moreover, that $R 1-R 4$ imply

$R 7 \quad[a]: a \in A . \supset . a=a+a$

Hence, $c f$. an analogous case in [4], we can conclude:

A dual associative Newman algebra can be considered as a semilattice with respect to the binary operation + to which the additional postulates are added concerning the properties of the operations $\times$ and - .

2 In [5], p. 418, an equational axiomatization of the mixed associative Newman algebras has been established. Analogously, we can define the dual mixed associative Newman algebras as follows:

Any algebraic structure

$$
\mathfrak{S}=\langle A,+, \times, \rightarrow\rangle
$$

where,$+ \times$ and $\rightarrow$ are three binary operations defined on the carrier set $A$, is a dual mixed associative Newman algebra, if it satisfies the following postulates:

$S 1 \quad[a b c]: a, b, c \in A . \supset . a+(b \times c)=(a+b) \times(a+c)$

S2 $[a b]: a, b \in A$. ว. $a+b=b+a$

S3 $[a b]: a, b \in A . \supset .(a \rightarrow b) \times(a+b)=b$

S4 $[a b c]: a, b, c \in A$.つ. $(a \rightarrow b)+(a+b)=c \rightarrow c$

Concerning the primitive binary operation $\rightarrow$ of the system $\mathfrak{S}$ it should be remarked that this operation is not a pseudo-complement operation $\Rightarrow$ which is a familiar primitive operation in the relatively pseudo-complemented lattices. It will be shown in section 3, point (2), below that H $^{2} 3$ verifies $S 1-S 4$, but falsifies a formula:

$$
[a b]: a, b \in A . \supset .(a \rightarrow(b \times c))+(a \rightarrow b)=a \rightarrow b
$$


which corresponds to the well-known formula of relatively pseudocomplemented lattices, $c f$. [1], p. 62:

$$
a \Rightarrow(b \times c) \leq a \Rightarrow b
$$

In section 3, point (3) below the mutual independency of the postulates S1-S4 will be proved. Again, using deductions entirely analogous to those given in [5], see p. 418, Theorem 2, we can establish that:

A dual mixed associative Newman algebra can be considered as a semi-lattice with respect to the primitive operation + to which the additional postulates are added concerning the properties of the primitive operations $\times$ and $\rightarrow$.

3 In order to establish the independencies which are announced in sections

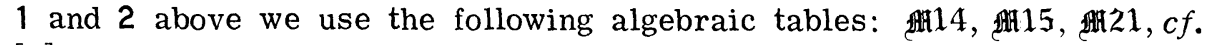
[6], p. 541 and p. 545, and:

晴2之

\begin{tabular}{c|ccc}
+ & $\alpha$ & 1 & 0 \\
\hline$\alpha$ & $\alpha$ & 1 & 0 \\
1 & 1 & 1 & 0 \\
0 & 0 & 0 & 0
\end{tabular}

\begin{tabular}{c|ccc}
$\times$ & $\alpha$ & 1 & 0 \\
\hline$\alpha$ & $\alpha$ & 1 & $\alpha$ \\
1 & 1 & 1 & 1 \\
0 & $\alpha$ & 1 & 0
\end{tabular}

\begin{tabular}{l|l}
$x$ & $-x$ \\
\hline$\alpha$ & 0 \\
1 & 0 \\
0 & 1
\end{tabular}

航3

\begin{tabular}{c|ll}
+ & 0 & $\eta$ \\
\hline 0 & 0 & 0 \\
$\eta$ & 0 & $\eta$
\end{tabular}

\begin{tabular}{c|cc}
$\times$ & 0 & $\eta$ \\
\hline 0 & 0 & $\eta$ \\
$\eta$ & $\eta$ & 0
\end{tabular}

\begin{tabular}{c|ll}
$\rightarrow$ & 0 & $\eta$ \\
\hline 0 & 0 & $\eta$ \\
$\eta$ & 0 & 0
\end{tabular}

佣 24

\begin{tabular}{c|ccccc}
+ & 0 & $\alpha$ & $\beta$ & $\gamma$ & $\delta$ \\
\hline 0 & 0 & 0 & 0 & 0 & 0 \\
$\alpha$ & 0 & $\alpha$ & 0 & 0 & $\alpha$ \\
$\beta$ & 0 & 0 & $\beta$ & 0 & $\beta$ \\
$\gamma$ & 0 & 0 & 0 & $\gamma$ & $\gamma$ \\
$\delta$ & 0 & $\alpha$ & $\beta$ & $\gamma$ & $\delta$
\end{tabular}

\begin{tabular}{c|ccccc}
$\times$ & 0 & $\alpha$ & $\beta$ & $\gamma$ & $\delta$ \\
\hline 0 & 0 & $\alpha$ & $\beta$ & $\gamma$ & $\delta$ \\
$\alpha$ & $\alpha$ & $\alpha$ & $\delta$ & $\delta$ & $\delta$ \\
$\beta$ & $\beta$ & $\delta$ & $\beta$ & $\delta$ & $\delta$ \\
$\gamma$ & $\gamma$ & $\delta$ & $\delta$ & $\gamma$ & $\delta$ \\
$\delta$ & $\delta$ & $\delta$ & $\delta$ & $\delta$ & $\delta$
\end{tabular}

航25

\begin{tabular}{c|ccccc}
$\rightarrow$ & 0 & $\alpha$ & $\beta$ & $\gamma$ & $\delta$ \\
\hline 0 & 0 & $\alpha$ & $\beta$ & $\gamma$ & $\delta$ \\
$\alpha$ & 0 & 0 & $\beta$ & $\gamma$ & $\beta$ \\
$\beta$ & 0 & $\alpha$ & 0 & $\gamma$ & $\gamma$ \\
$\gamma$ & 0 & $\alpha$ & $\beta$ & 0 & $\alpha$ \\
$\delta$ & 0 & 0 & 0 & 0 & 0
\end{tabular}

\begin{tabular}{c|ccc}
+ & $\alpha$ & 1 & 0 \\
\hline$\alpha$ & $\alpha$ & 1 & 0 \\
1 & $\alpha$ & 1 & 0 \\
0 & 0 & 0 & 0
\end{tabular}

\begin{tabular}{c|ccc}
$\times$ & $\alpha$ & 1 & 0 \\
\hline$\alpha$ & $\alpha$ & 1 & $\alpha$ \\
1 & 1 & 1 & 1 \\
0 & $\alpha$ & 1 & 0
\end{tabular}

\begin{tabular}{c|ccc}
$\rightarrow$ & $\alpha$ & 1 & 0 \\
\hline$\alpha$ & 0 & 0 & 0 \\
1 & 0 & 0 & 0 \\
0 & $\alpha$ & 1 & 0
\end{tabular}




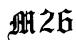

\begin{tabular}{c|cc}
+ & 0 & $\alpha$ \\
\hline 0 & 0 & 0 \\
$\alpha$ & 0 & 0
\end{tabular}

\begin{tabular}{c|cc}
$\times$ & 0 & $\alpha$ \\
\hline 0 & 0 & $\alpha$ \\
$\alpha$ & $\alpha$ & 0
\end{tabular}

\begin{tabular}{c|cc}
$\rightarrow$ & 0 & $\alpha$ \\
\hline 0 & 0 & 0 \\
$\alpha$ & 0 & 0
\end{tabular}

明27

\begin{tabular}{c|llll}
+ & 0 & $\alpha$ & $\beta$ & $\gamma$ \\
\hline 0 & 0 & 0 & 0 & 0 \\
$\alpha$ & 0 & $\alpha$ & $\gamma$ & $\beta$ \\
$\beta$ & 0 & $\gamma$ & $\beta$ & $\alpha$ \\
$\gamma$ & 0 & $\beta$ & $\alpha$ & $\gamma$
\end{tabular}

\begin{tabular}{c|cccc}
$\times$ & 0 & $\alpha$ & $\beta$ & $\gamma$ \\
\hline 0 & 0 & $\alpha$ & $\beta$ & $\gamma$ \\
$\alpha$ & $\alpha$ & 0 & $\gamma$ & $\beta$ \\
$\beta$ & $\beta$ & $\gamma$ & 0 & $\alpha$ \\
$\gamma$ & $\gamma$ & $\beta$ & $\alpha$ & 0
\end{tabular}

\begin{tabular}{c|cccc}
$\rightarrow$ & 0 & $\alpha$ & $\beta$ & $\gamma$ \\
\hline 0 & 0 & $\alpha$ & $\beta$ & $\gamma$ \\
$\alpha$ & 0 & 0 & $\alpha$ & $\alpha$ \\
$\beta$ & 0 & $\beta$ & 0 & $\beta$ \\
$\gamma$ & 0 & $\gamma$ & $\gamma$ & 0
\end{tabular}

(1) Since: (a) 915 verifies $R 2, R 3$ and $R 4$, but falsifies $R 1, c f$. [6], p. 542;

(b) 明2 verifies $R 1, R 3$ and $R 4$, but flasifies $R 2$ for $a / \alpha$ and $b / 1$ : (i) $\alpha=\alpha$, (ii) $\alpha+(1 \times-1)=\alpha+(1 \times 0)=\alpha+0=0$; (c) 14 verifies $R 1, R 2$ and $R 4$, but falsifies $R 3$ for $a / \gamma, b / \alpha$ and $c / \beta$ : (i) $\gamma+(\alpha \times \beta)=\gamma+1=\gamma$, (ii) $(\beta+\gamma) \times$ $(\alpha+\gamma)=\beta \times 0=0$; and (d) An $_{2}$ verifies $R 1, R 2$ and $R 3$, but falsifies $R 4$, $c f$. [6], p. 545, the proof that the axioms $R 1-R 4$ are mutually independent is complete.

(2) Since An $_{23}$ verifies $S 1, S 2, S 3$ and $S 4$, but falsifies the formula $(\beta)$ for $a / 0, b / \eta$ and $c / \eta:($ i) $(0 \rightarrow(\eta \times \eta)+(0 \rightarrow \eta)=(0 \rightarrow 0)+\eta=0+\eta=0$, (ii) $0 \rightarrow$ $\eta=\eta$, we know that $(\beta)$ is not a consequence of $S 1-S 4$.

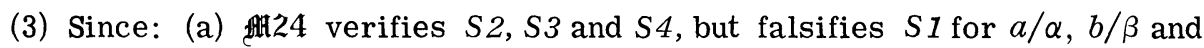
$c / \gamma$ : (i) $\alpha+(\beta \times \gamma)=\alpha+\delta=\alpha$, (ii) $(\alpha+\beta) \times(\alpha+\gamma)=0 \times 0=0$; (b) 1925 verifies $S 1, S 3$ and $S 4$, but falsifies $S 2$ for $a / \alpha$ and $b / 1$ : (i) $\alpha+1=1$, (ii) $1+\alpha=$ $\alpha$; (c) An $_{2} \mathfrak{b}$ verifies $S 1, S 2$ and $S 4$, but falsifies $S 3$ for $a / 0$ and $b / \alpha$ : (i) ( $O \rightarrow$ $\alpha) \times(0+\alpha)=0 \times 0=0$, (ii) $\alpha=\alpha$; and (d) falsifies $S 4$ for $a / \alpha, b / \beta$ and $c / 0$ : (i) $(\alpha \rightarrow \beta)+(\alpha+\beta)=\alpha+\gamma=\beta$, (ii) $0 \rightarrow$ $\bar{O}=0$, the proof that the axioms S1-S4 are mutually independent is complete.

\section{Corrections:}

(A) The proof of $F 3$ in [3], p. 268, lines 8-13, contains rather bad misprints. It should be given, as follows:

F3 $[a b]: a, b \in A . \supset . a=(b+\bar{b}) \times a$

PR $[a b]: \operatorname{Hp}(1) . \supset$.

$$
\begin{array}{rr}
a & =a \times(b+\bar{b})=(\bar{b} \times a)+(b \times a)=((\bar{b} \times(b+\bar{b})) \times a)+((b \times(b+\bar{b})) \times a) \\
& =(\bar{b} \times((b+\bar{b}) \times a))+(b \times((b+\bar{b}) \times a)) \\
{[1 ; F 2 ; H 1 ; F 2]} \\
=((b+\bar{b}) \times a) \times(b+\bar{b})=(b+\bar{b}) \times a & {[A 10 ; L 1]} \\
{[H 1 ; F 2]}
\end{array}
$$

(B) Since H5, $c f$. [2], p. 263, falsifies $H 1$ for $a / 0, b / \alpha$ and $c / 1$ : (i) $0+$ $(\alpha \times 1)=0+1=1$, (ii) $(1+0) \times(\alpha+0)=1 \times \alpha=\alpha$, the statement “明 5 verifies $F 1$ and $H 1$, but falsifies $F 2$," which due to a manuscript mix-up appeared in [3], p. 268, lines $30-31$, is obviously false. It should be substituted by the correct one:

"an algebraic table 
明28

\begin{tabular}{c|ccc}
+ & $\alpha$ & 1 & 0 \\
\hline$\alpha$ & $\alpha$ & 1 & $\alpha$ \\
1 & 1 & 1 & 1 \\
0 & $\alpha$ & 1 & 0
\end{tabular}

\begin{tabular}{c|ccc}
$\times$ & $\alpha$ & 1 & 0 \\
\hline$\alpha$ & $\alpha$ & 1 & 0 \\
1 & 1 & 1 & 0 \\
0 & 0 & 0 & 0
\end{tabular}

\begin{tabular}{c|c}
$x$ & $-x$ \\
\hline$\alpha$ & 0 \\
1 & 0 \\
0 & 1
\end{tabular}

verifies $F 1, H 1$ and $L 1$, but falsifies $F 2$ for $a / \alpha$, and $b / 1$ : (i) $\alpha=\alpha$, (ii) $\alpha \times$ $\left((1+\overline{1})=\alpha \times(1+0)=\alpha \times 1=1{ }^{\prime}\right.$.

\section{REFERENCES}

[1] Rasiowa, H., and R. Sikorski, "The mathematics of metamathematics," Monografie Matematyczne, tom 41. Państwowe Wydawnictwo Naukowe, Warszawa (1963).

[2] Sobociński, B., "A new formalization of Newman algebras," Notre Dame Journal of Formal Logic, vol. XIII (1972), pp. 255-264.

[3] Sobociński, B., "An equational axiomatization of associative Newman algebras," Notre Dame Journal of Formal Logic, vol. XIII (1972), pp. 265-269.

[4] Sobociński, B., "A semi-lattice theoretical characterization of associative Newman algebras," Notre Dame Journal of Formal Logic, vol. XIII (1972), pp. 283-285.

[5] Sobociński, B., "An equational axiomatization and a semi-lattice theoretical characterization of mixed associative Newman algebras," Notre Dame Journal of Formal Logic, vol. XIII (1972), pp. 407-423.

[6] Sobociński, B., "Solution to the problem concerning the Boolean bases for cylindric algebras," Notre Dame Journal of Formal Logic, vol. XIII (1972), pp. 529-545.

University of Notre Dame

Notre Dame, Indiana 